\title{
Proposing a New Lemma about Odd Numbers and a New Conjecture about a Sequence of Prime Numbers
}

\author{
Salman Mahmud* \\ Student of BIAM Model School and College, Bogura, Bangladesh. \\ *Corresponding Author: Salman Mahmud, Student of BIAM Model School and College, Bogura, \\ Bangladesh.
}

Abstract: Here in this article firstly I will prove a new lemma and the lemma is, every odd number which is not a multiple of 3 and greater than 1 can be represented as $\sqrt{2^{2+n} \cdot 3 k+1}$ where $n$ and $k$ are positive integers. Then I will propose a new conjecture about a sequence of prime numbers.

Keywords: Number theory, odd numbers, multiple of three, positive integers, prime numbers, conjecture, lemma.

\section{INTRODUCTION}

In mathematics, informal logic and argument mapping, a lemma is a generally minor, proven proposition which is used as a stepping stone to a larger result. For that reason, it is also known as a "helping theorem" or an "auxiliary theorem". Here I have proven a new lemma and the lemma is, every odd number which is not a multiple of 3 and greater than 1 can be represented as $\sqrt{2^{2+n} \cdot 3 k+1}$ where $\mathrm{n}$ and $\mathrm{k}$ are positive integers. To prove this lemma we have to use some theorems about odd numbers. Again, a conjecture is a conclusion or a proposition which is suspected to be true due to preliminary supporting evidence, but for which no proof or disproof has yet been found. Here I will propose a new conjecture about a sequence of prime numbers.

\section{THE NEW LEMMA}

The lemma is as follows,

Lemma 1: "Every odd number which is not a multiple of 3 and greater than 1 can be represented as $\sqrt{2^{2+n} \cdot 3 k+1}$ where $\mathrm{n}$ and $\mathrm{k}$ are positive integers."

The theorem which is needed to prove this lemma,

Theorem 1: "The square of odd numbers minus 1 is divisible by $8 . "$

The square of multiples of 3 minus 1 is never divisible by 3 because the square of any multiple of 3 is also a multiple of 3 and after subtracting 1 it will be an even number. This even number won't be divisible by 3 . To prove the lemma we have to prove that the square of any number which is not a multiple of 3 minus 1 is divisible by $3 \mathrm{k}$.

Now, we can represent every number ( not the multiples of 3 ) by using the multiples of 3 . Every number can be represented as $3 k+1$ (1) or $3 k+2$ (2) or $3 k-1$ (3)or $3 k-2$ (4).

Now, squaring the numbers which are not the multiples of 3 and subtracting 1 we get,

$$
\begin{aligned}
& (3 k+1)^{2}-1=9 k^{2}+6 k+1-1=3 k(3 k+2) \\
& (3 k+2)^{2}-1=9 k^{2}+12 k+4-1 \\
& =3\left(3 k^{2}+4 k+1\right) \\
& (3 k-1)^{2}-1=9 k^{2}-6 k+1-1=3 k(3 k-2) \\
& (3 k-2)^{2}-1=9 k^{2}-12 k+4-1=3\left(3 k^{2}-4 k+1\right)
\end{aligned}
$$


Here we can see that $3 k(3 k+2)$ and $3 k(3 k-2)$ are divisible by $3 k$. Again, $3\left(3 k^{2}+4 k+1\right)$ and $3\left(3 k^{2}-4 k+1\right)$ are divisible by 3 . In this case $\mathrm{k}=1$. So we can say the square of any number which is not a multiple of 3 minus 1 is divisible by $3 \mathrm{k}$. Again, The square of odd numbers minus 1 is divisible by 8 or $2^{3}$. That's why, the square of every odd number which is not a multiple of 3 minus 1 is divisible by 3 and $2^{3}$. The square of 1 minus 1 is 0 and 0 is divisible by every number. So we have to take all the odd numbers greater than 1 . Now look at the table below,

\begin{tabular}{|c|c|c|}
\hline Odd Numbers & $\begin{array}{l}\text { The square of the odd numbers } \\
\text { minus } 1\end{array}$ & Divisible by \\
\hline 3 & $3^{2}-1=8$ & $2^{3}$ \\
\hline 7 & $7^{2}-1=48$ & $2^{4}$ \\
\hline 23 & $23^{2}-1=528$ & $2^{4}$ \\
\hline 31 & $31^{2}-1=960$ & $2^{6}$ \\
\hline
\end{tabular}

Now we can write,

The square of odd numbers minus 1 is divisible by at least 8 . But it can be divisible by $2^{4}, 2^{5}, 2^{6}$ etc. So we can write, The square of odd numbers minus 1 is divisible by $2^{2+n}$ where $\mathrm{n}$ is any positive integer.

Now we get,

(1) The square of any number which is not a multiple of 3 minus 1 is divisible by $3 \mathrm{k}$.

(2) The square of odd numbers minus 1 is divisible by $2^{2+n}$ where $\mathrm{n}$ is any positive integer.

Combining (1) and (2) we can write, the square of any odd number which is not a multiple of 3 minus 1 is divisible by $2^{2+n} \cdot 3 k$. So we can represent the odd number which is not a multiple of 3 and greater than 1 as $\sqrt{2^{2+n} \cdot 3 k+1}$. For example $5=\sqrt{2^{2+1} \cdot 3 \cdot 1+1}, 7=\sqrt{2^{2+2} \cdot 3 \cdot 1+1}$ etc.

\section{The CONJECTURE}

The conjecture is as follows,

"Suppose, set $S=\{10,20,30,40, \ldots \ldots\}$. If there are three or four prime numbers between $n_{a}$ and $\left(n_{a}+10\right)$ and also between $n_{b}$ and $\left(n_{b}+10\right)$ where $n_{a}, n_{b} \in S$ and $n_{a}>n_{b}$ or $n_{a}<n_{b}$, then the difference between $n_{a}$ and $n_{b}\left(n_{a} \sim n_{b}\right)$ will be a multiple of 30 ."

For example:

There are four prime numbers $(11,13,17,19)$ between 10 and $(10+10)$ or 20 and three prime numbers $(41,43,47)$ between 40 and $(40+10)$ or 50 . Here in this case $n_{a}=10$ and $n_{b}=40$. So, the difference between $n_{a}$ and $n_{b}$ is 30 which is a multiple of 30 .

Again, There are three prime numbers $(71,73,79)$ between 70 and $(70+10)$ or 80 , three prime numbers $(223,227,229)$ between 220 and $(220+10)$ or 230 and three prime numbers $(311,313,317)$ between 310 and $(310+10)$ or 320 . So, the difference between 70 and 220 is 150 or $(30 \times 5)$ which is a multiple of 30. Again the difference between 220 and 310 is 90 and the difference between 70 and 310 is 240 . Here 90 and 240 are also the multiples of 30.Here we can give thousands of example of this conjecture because we have tried this by all the prime numbers from 1 to 100000 . But we don't have any proof of this conjecture.

\section{CONCLuSion}

The lemma is interesting and can be very important for number theory. The conjecture is still unsolved. Anybody can prove or disprove it.

\section{ACKNOWLEDGEMENTS}

I should Thanks MD. Shah Alam and MST. Sabina Yesmin for providing me with the help materials.

\section{REFERENCES}

[1] Math is Fun n. d. Prime Number List, viewed 22 April 2020, http://www.mathisfun.com/numbers/primenumbers-to-10k.html 
Proposing a New Lemma about Odd Numbers and a New Conjecture about a Sequence of Prime Numbers

[2] Wikipedia n. d. Lemma (mathematics), viewed 9 July 2020, http://en.m.wikipedia.org/wiki/lemma_ (mathematics)

[3] Wikipedia n. d. Conjecture, viewed 9 July 2020, http://en.m.wikipedia.org/wiki/conjecture

[4] Proofs from the book n. d. Square odd numbers minus 1, viewed 9 July 2020, http://proofsfromthebook.com /2014/06/10/square-odd-numbers-minus-1/

\section{AUTHOR'S BIOGRAPHY}

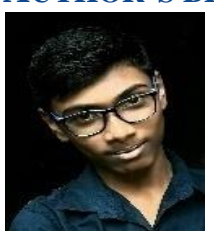

Salman Mahmud, studying at BIAM Model School and College, Bogura, Bangladesh. My core research interest is in modern physics and pure mathematics. I like to spend time with my father (MD. Shah Alam) and my mother (MST. Sabina Yesmin). I also like to quarrel with my younger brother (Suyaib Sadik)!

Citation: Salman Mahmud, Proposing a New Lemma about Odd Numbers and a New Conjecture about a Sequence of Prime Numbers, International Journal of Scientific and Innovative Mathematical Research (IJSIMR), vol. 8, no. 3, pp. 6-8, 2020. Available : DOI: http://dx.doi.org/10.20431/2347-3142.0803003

Copyright: (c) 2020 Authors. This is an open-access article distributed under the terms of the Creative Commons Attribution License, which permits unrestricted use, distribution, and reproduction in any medium, provided the original author and source are credited. 